Regular Article

\title{
A metallic glass syntactic foam with enhanced energy absorption performance
}

\author{
H. Lin ${ }^{\text {a }}$, H.Y. Wang ${ }^{\text {a }}$, C. Lu ${ }^{\text {b }}$, L.H. Dai ${ }^{\text {a,* }}$ \\ a State Key Laboratory of Nonlinear Mechanics, Institute of Mechanics, Chinese Academy of Sciences, Beijing 100190, China \\ b Department of Mechanical Engineering, Curtin University, Perth, WA 6845, Australia
}

\section{A R T I C L E I N F O}

\section{Article history:}

Received 13 March 2016

Received in revised form 31 March 2016

Accepted 31 March 2016

Available online 11 April 2016

\section{Keywords:}

Bulk metallic glass

Syntactic foam

Energy absorption

Shear bands

\begin{abstract}
A B S T R A C T
By using the high-pressure melt infiltration technique, a syntactic foam is fabricated with bulk metallic glass and alumina cenospheres. Compared to pure metallic glass foams, the new foam possesses a greatly enhanced energy absorbing capacity of $113.6 \mathrm{MJ} \mathrm{m}^{-3}$ due to the combination of high strength, stability and ductility. It is shown that the high strength of the foam primarily results from alumina cenospheres, which enhance its stability and induce a stable stress platform. Both the collapse of struts and multiple shear bands in metallic glass matrix accommodate large deformation.
\end{abstract}

(c) 2016 Elsevier Ltd. All rights reserved.
Metallic foams are recognized as attractive structural and functional materials for their unique combination of mechanical, physical and chemical properties such as high density-compensated strength, mechanical energy absorption and acoustic damping [1-4]. The increasing interest and importance in structural applications have motivated investigation of bulk metallic glasses (BMGs) as alternative materials of these foams on account of their ultrahigh strength, exceptional elasticity, excellent corrosion resistance, and process ability [5-9]. Therefore, considerable efforts on BMG foams with high strength and ductility have shown that their energy absorption capability is much better than those of pure $\mathrm{Al}$ and its alloy foams [10-17]. However, there are still some limitations in the application of metallic glass foams mainly because the existing preparation methods are too complicated to control. For example, to avoid heterogeneous nucleation leading to crystallization that reduces the strength of metallic glass matrix, $\mathrm{BaF}_{2}$ particles with high melting point and thermal stability were used as placeholders $[6,17]$. Similarly, foaming by blowing agents is limited in some precious metals such as Pd-based metallic glasses [12-14]. In addition, compared with syntactic $\mathrm{Al}$ and high strength alloy foams, the existing metallic glass foams have no significant advantage in energy absorption [18-22]. Fortunately, it is shown that the addition of non-metallic such as brittle ceramic particles and fibers is an effective way to produce materials with attractive engineering attributes [23-26]. The high content of brittle second phases can increase both the strength and ductility of composites $[25,26]$. In this paper, using hollow ceramic microspheres as a reinforcer that are also effective in avoiding crystallization, we

\footnotetext{
* Corresponding author.

E-mail address: lhdai@Inm.imech.ac.cn (L.H. Dai).
}

fabricate a BMG syntactic foam through pressure infiltration, which was previously applied to make metal matrix composites with monolithic (non-hollow) ceramic reinforcement [27]. The results show that the combination of high strength and ductility of BMG syntactic foams leads to an enhanced energy absorption capacity, which is much higher than those of available BMG foams [10-20].

We chose the $\mathrm{Zr}_{41.25} \mathrm{Ti}_{13.75} \mathrm{Cu}_{12.5} \mathrm{Ni}_{10} \mathrm{Be}_{22.5}$ system (Vit 1 ) as the parent material because of its high processability and fragility $[28,29]$. Alumina cenospheres with the average size of $500-600 \mu \mathrm{m}$ and a relative wall thickness of $t / R=0.13$ were selected as placeholder particles. To prevent crystallization during infiltration casting, a material preparation device combining quick melting, pressure seepage and rapid cooling was invented. Master alloy button of Vit 1 was acquired by arc-melting the high-purity (R99.5\%) constituent elements several times under a Ti-gettered Ar atmosphere. Then, the Vit 1 ingot was placed on the top of a graphite die that was filled with alumina cenospheres. Both the ingot and graphite die were picked up in a quartz tube sealed with a solenoid valve, which can be easily connected with a diffusion vacuum pump or an argon gas bomb. Initially, the solenoid valve was connected with the diffusion vacuum pump until vacuum in the quartz tube reached up to $6 \mathrm{MPa}$. Then, the master ingot was heated by an induction coil surrounding the quartz tube. After the master alloy was melted, the solenoid valve was connected with the argon gas bomb, and metal liquid was pressed into the graphite die to fill the space among alumina cenospheres. Almost at the same time, the tube was sub-merged by the mixture of saturated $\mathrm{NaCl}$ solution and ice cubes through a U-turn tube and cooled down quickly. For comparison, the pure Vit 1 foam with similar apertures was prepared by a similar method just replacing alumina cenospheres with soluble $\mathrm{NaCl}$ 

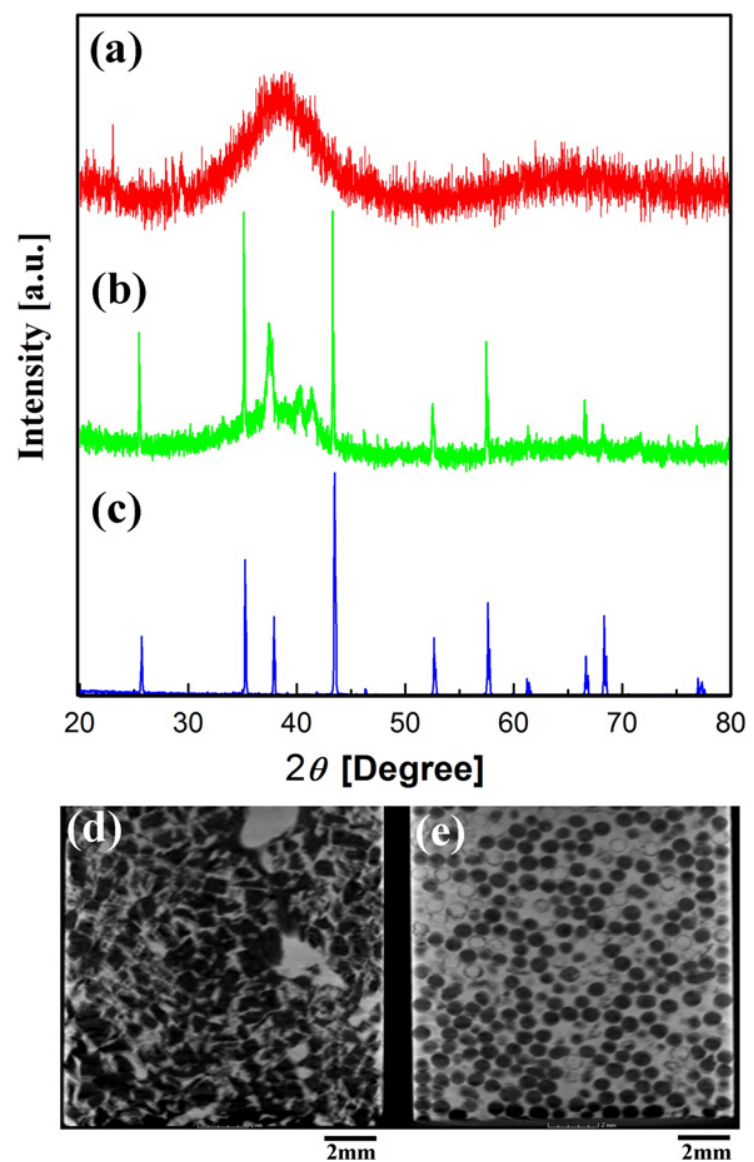

Fig. 1. (a) - (c) XRD patterns of Vit 1 foam, BMG syntactic foam and alumina powder; (d) - (e) computerized tomographies of Vit 1 and BMG syntactic foams.

placeholders, and dissolved salt particles by immersion in distilled water [16].

The amorphous phase nature of foam samples was analyzed by using X-ray diffraction (XRD) with $\mathrm{Cu}-\mathrm{K} \alpha$ radiation (M18AHF). As seen from the XRD patterns in Fig. 1(a)-(c), the structures of Vit 1 and BMG syntactic foams are amorphous. The volume fraction $V_{f}$ of cenospheres in the syntactic foam can be calculated by using the ruleof-mixture, that is

$V_{f}=\frac{\rho_{s}-M / V}{\rho_{s}-\rho_{c}}$

where $\rho_{s}$ is the density of BMG matrix, $M / V$ is the density of syntactic foams with $M$ the mass and $V$ the volume of a foam, and $\rho_{c}$ is the density of cenospheres. Based on measurements, the density of Vit 1 is $6.0 \mathrm{~g} \mathrm{~cm}^{-3}$, the apparent density of BMG syntactic foams is $3.2 \mathrm{~g} \mathrm{~cm}^{-3}$, and the density of alumina hollow spheres with an average size of $500-600 \mu \mathrm{m}$ is $1.4 \mathrm{~g} \mathrm{~cm}^{-3}$. Thus, the volume fraction of cenospheres in syntactic foams can be obtained as $59.7 \%$. The porosity of pure Vit 1 foam was about $67 \%$. Then, the samples were scanned with the X-ray computerized tomography, and the images are shown in Fig. 1(d)-(e). The detailed geometry dimensions were also measured based on the image of BMG syntactic foams. The average diameter $d$ of spherical pores in the BMG syntactic foam and the average center distance $l$ between spherical pores are 567 and $735 \mu \mathrm{m}$, respectively.

Quasi-static uniaxial compression tests with a strain rate of $10^{-3} \mathrm{~s}^{-1}$ were performed on the MTS-810 material test system at ambient temperature. Cylinder specimens with a diameter of $10 \mathrm{~mm}$ made of pure Vit 1 and BMG syntactic foams were prepared by a diamond grinding wheel and a diamond wafering saw. Strain was calculated from the crosshead displacement, and corrected to take into account deflection
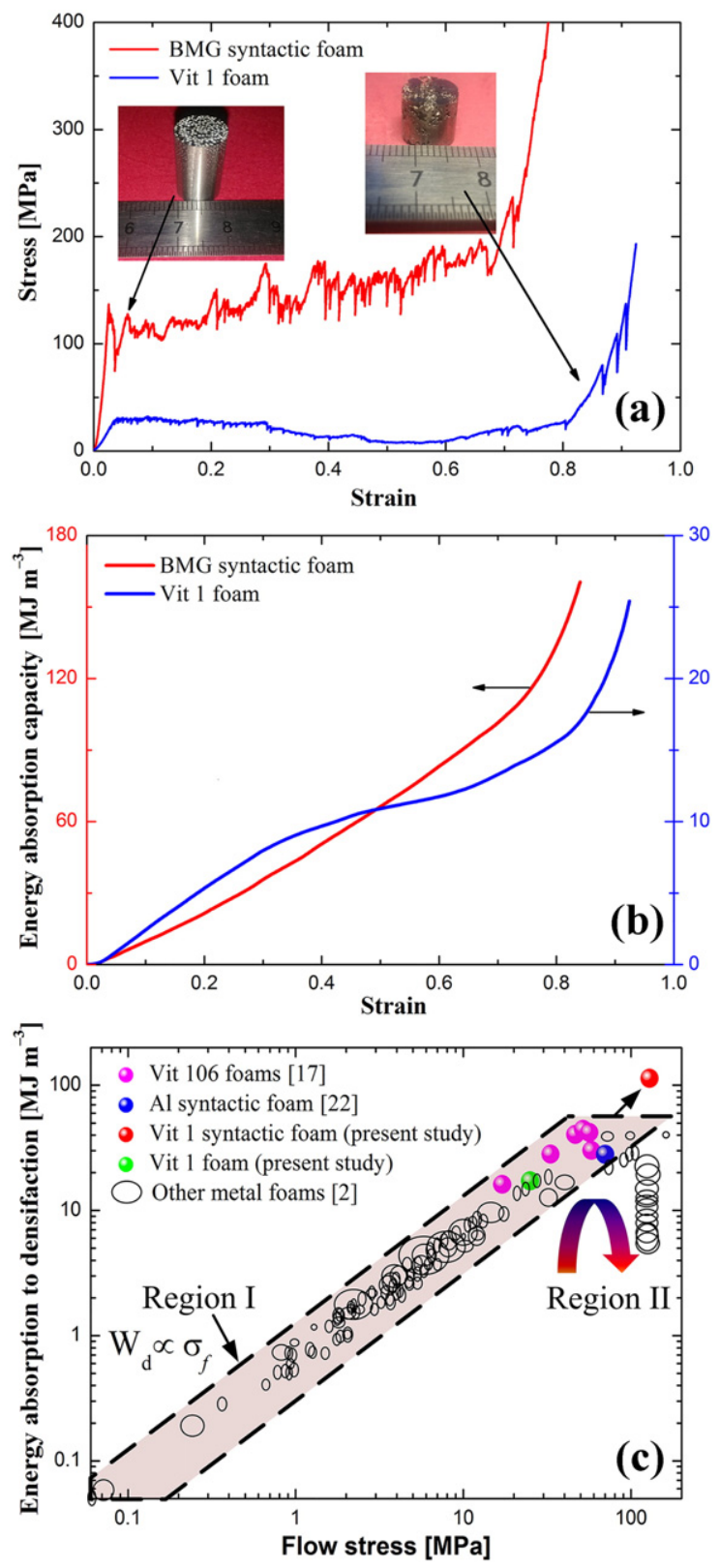

Fig. 2. Quasi-static compression tests at the strain rate of $1 \times 10^{-2} \mathrm{~s}^{-1}$ : (a) stress-strain curves of two foams and (b) their corresponding EAC as a function of strain. (c) The log$\log$ plot of EAC per unit volume to densification as a function of flow stress at $25 \%$ strain for different foams. In region I between two dashed lines, EAC to densification is proportional to flow stress and in region II, EAC to densification drops quickly.

of the load frame. As shown in Fig. 2(a), it is obvious that the BMG syntactic foam exhibits an initial linear stress-strain response until a stress peak appears at strain of about $2.5 \%$. Here, we define the first peak stress on a compressive stress-strain curve as strength $\sigma_{y}$ of the foam. Thus, the strength of the BMG syntactic foam is $137 \mathrm{MPa}$. After the linear deformation stage, there is a long stress plateau with numerous flow serrations that can be attributed to a sudden high elastic energy release. Beyond the deformation plateau, stress drastically increases as strain reaches about $75 \%$, which indicates the occurrence of densification with the strain of $\varepsilon_{d}$. To represent the stability during a deformation process, we define the "strain-hardening" index as

$n=\frac{\sigma_{p l}-\sigma_{y}}{\sigma_{y}}$

where $\sigma_{p l}$ is the average plateau stress over the plastic flow region, defined as $\sigma_{p l}=\int_{\varepsilon_{y}}^{\varepsilon_{d}} \sigma \mathrm{d} \varepsilon /\left(\varepsilon_{d}-\varepsilon_{y}\right)$ [30], and $\varepsilon_{y}$ is the strain corresponding 

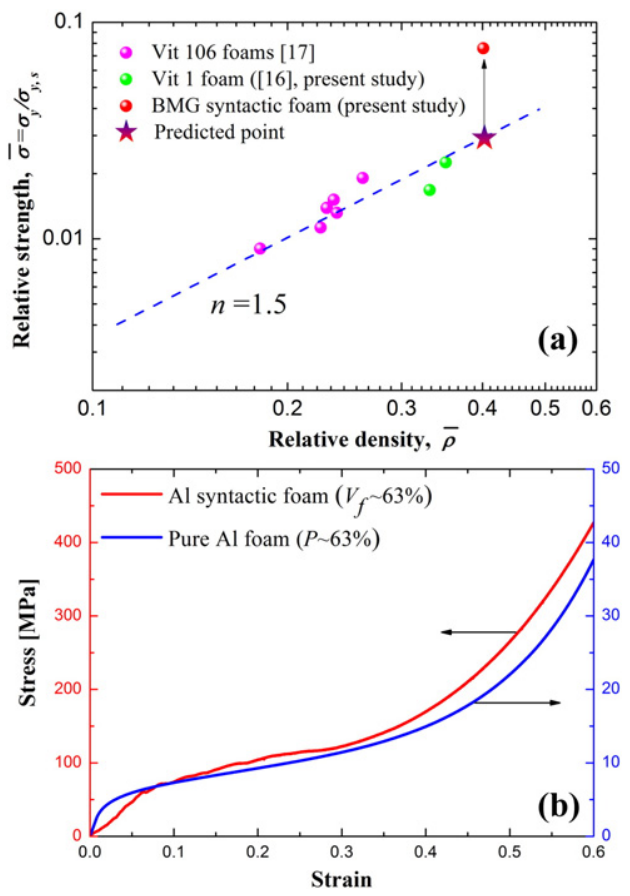

Fig. 3. (a) Normalized compressive strength for three different kinds of BMG foams as a function of the relative density. (b) Stress-strain curves of pure and syntactic Al foams at the strain rate of $1 \times 10^{-2} \mathrm{~s}^{-1}$.

to the strength $\sigma_{y}$. Then, we can obtain that the index $n$ is about 0.15 for the BMG syntactic foam, which implies that the material possesses an obvious strain-hardening ability. As comparison, a stress-strain curve of the pure open-cell Vit 1 foam was also given in Fig. 2(a). Similarly, its strength can be obtained as $\sim 30 \mathrm{MPa}$, densification strain as $\sim 85 \%$, and "strain-hardening" index as -0.35 . Here, it is worth noting that a negative index usually indicates that a material is unstable during deformation. Actually, such negative indexes are commonly reported in previous studies on pure BMG foams [11,12]. Thus, the transition of index $n$ from negative to positive shows that the addition of alumina cenospheres can enhance the foam's stability through inducing a stable stress platform.

Based on these stress-strain curves, we can further examine the energy absorption capacity (EAC), which is calculated by the area under a stress-strain curve, that is

$W=\int_{0}^{\varepsilon} \sigma \mathrm{d} \varepsilon$

As shown in Fig. 2(b), the EAC of BMG syntactic foams is much higher than that of Vit 1 foams during the loading history. Furthermore, Fig. 2(c) summarizes the EACs of currently available metal [2], Al cenosphere [22], Vit 106 [17], Vit 1 and BMG syntactic foams, at the densification strain, as a function of flow stress at $25 \%$ strain. It is seen that the EAC to densification is proportional to flow stress between the two dashed lines (region I). In region II, the yield stress that is above a bottleneck stress of 50-60 MPa drops quickly because high strength can usually be acquired at the cost of reduction of porosity that is proportional to the densification strain. However, BMG syntactic foams may provide one of promising methods for breaking through this barrier. Thus, a deep understanding on their enhancing mechanism can give insights into the development of high EAC materials.

One main factor that contributes to high EAC of BMG syntactic foams is their enhanced strength. Fig. 3 shows the normalized strength data of BMG syntactic and pure Vit 1 foams ([16] and present study) and Vit 106 foams [17]. Here, the relative density $\rho$ (the horizontal axis) is defined as, $\rho=1-V_{f}$, for BMG syntactic foams to eliminate the effect of alumina cenospheres. The compressive strength of BMG matrix, $\sigma_{y, s}=1800 \mathrm{MPa}$, for Vit 106 and Vit 1 [16,31] were used to normalize
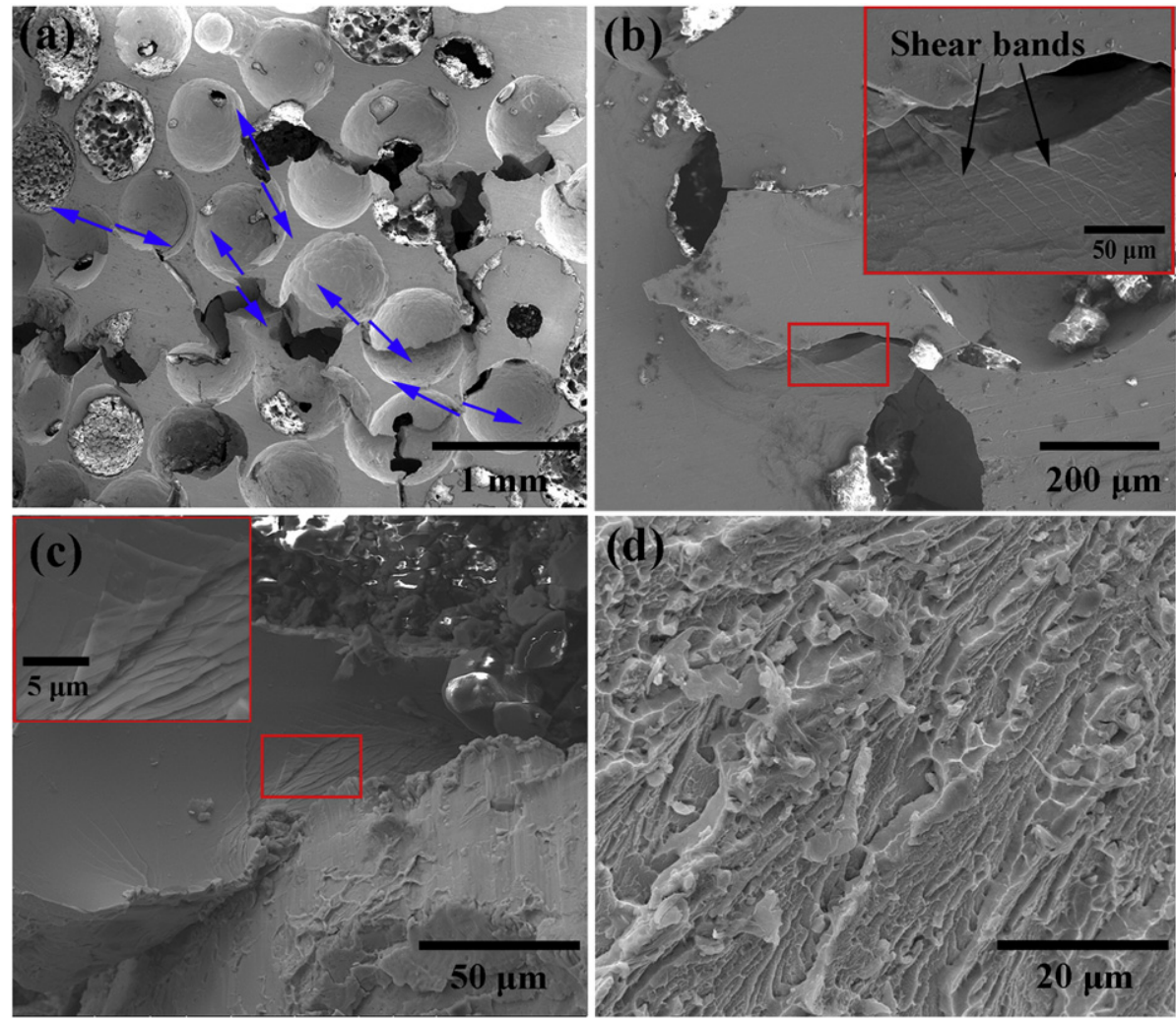

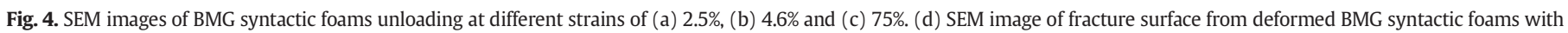
dimple structures. 
the strength of BMG foams. Based on a large number of empirical data [1], the strength $\sigma_{y}$ exhibits a power-law scaling behavior, which can be represented as

$\frac{\sigma_{y}}{\sigma_{y, s}}=C(1-P)^{n}$,

where $P$ is the porosity of a foam. As shown in Fig. 3(a), this relationship holds for open-cell BMG foams within a large density range and with the power law index of about 1.5 , which is consistent with the analysis proposed by Gibson and Ashby [1], simplifying foams as a unit cell and assuming plastic hinge formation at nodes. Obviously, the strength of BMG syntactic foams is about $80 \mathrm{MPa}$ higher than that of open-cell BMG foams with the same porosity predicted by Eq. (4). To elucidate the strengthening mechanism, pure and syntactic Al foams with the same porosity were prepared and their stress-strain curves are shown in Fig. 3(b). In contrast to that of pure Al foam, the raised strength of syntactic $\mathrm{Al}$ foam is about $74 \mathrm{MPa}$, which is very close to $80 \mathrm{MPa}$, indicating that strengthening is independent of metal matrix and primarily results from the addition of alumina cenospheres [32]. In addition, closed-cell structures and round pores play a complementary role in minimizing the loss of strength associated with stress concentrations [17].

The other main factor is their ductility or the length of a stress platform. To systemically investigate the deformation process, the scanning electron microscope (SEM) observations with interrupted uniaxial compression tests were carried out. As shown in Fig. 4(a), observation was firstly conducted at a strain of about $2.5 \%$, where the specimen just suffered a stress drop. It is observed that shear fracture, as marked with blue arrows, occurs in the struts. Here, it is worth noting that the average size $(\sim 170 \mu \mathrm{m})$ of struts is much larger than the plastic zone size $(\sim 60 \mu \mathrm{m})$ for $\mathrm{Zr}$-based BMGs, through which the stable crack growth happens inside a dominant shear band [33]. Fortunately, unlike catastrophic brittle fracture in monophase BMGs, shear bands in BMG syntactic foams correspond to local unstable slips that can be stopped by alumina cenospheres, leading to a non-zero sticking stress. As loading continues, crushed struts contact (see Fig. 4(b)), which leads to elastic reloading until stress exceeds the strength. Then, a subsequent stress drop occurs and stress redistributes in the whole sample. However, the loading state becomes much more complicated than the initial one. The inset in Fig. 4(b) presents the detail of a broken strut, where multiple shear bands formed under bending load that ensures a relatively stable deformation. Elastic reloading and burst unloading accompanied with the stress redistribution alternatively repeat until all pores are compacted, during which alumina fragments completely fill the interspace of crushed cells and transfer stress among the collapsed struts. Actually, alumina fragments possess the high strength (1154 MPa) [22] and effectively compensate for the loss of hardening in the plastic deformation of BMGs [34]. Fig. 4(c) shows the interruption at a stain of about 75\%, which is close to densification (see the inset for details). The high density of shear bands indicates that the matrix has also accommodated a considerable deformation besides the collapse of struts. As shown in Fig. 4(d), the dimple structure on fracture surface of broken struts is an indication of ductility [35], illustrating that the struts fracture plastically.
In summary, we have developed a new material (i.e., the BMG/alumina cenosphere syntactic foam) and proved that alumina cenospheres as reinforcement are feasible to avoid crystallization. In contrast to other methods, the fabrication procedure is simple with low cost. Moreover, it is shown that the syntactic foam performs better in energy absorption than metallic glass and traditional metal foams, which is due to the optimal combination of high strength, stability and ductility.

\section{Acknowledgments}

The work is supported by the National Natural Science Foundation of China (Grant Nos. 11172322, 11472287, 11572324, and 11202221), the National Basic Research Program of China (Grant No. 2012CB937500), and the CAS/SAFEA International Partnership Program for Creative Research Teams.

\section{References}

[1] L.J. Gibson, M.F. Ashby, Cellular Solids: Structure and Properties, second ed. Cambridge University Press, Cambridge, 1997.

[2] M.F. Ashby, A.G. Evans, N.A. Fleck, L.J. Gibson, J.W. Hutchinson, H.N.G. Wadley, Metal Foams: A Design Guide, Butterworth-Heinemann, Boston, 2000.

[3] E. Amsterdam, J.T.M. De Hosson, P.R. Onck, Scr. Mater. 59 (2008) 653.

[4] H.P. Degischer, B. Kriszt, Handbook of Cellular Metals, Weinheim, Wiley-VCH, 2002

[5] M.F. Ashby, A.L. Greer, Scr. Mater. 54 (2006) 321.

[6] A.H. Brothers, D.C. Dunand, Adv. Mater. 17 (2005) 484.

[7] O.V. Kuzmin, Y.T. Pei, J.T.M. De Hosson, Appl. Phys. Lett. 98 (2011) 233104.

[8] Z.D. Zhu, P. Jia, J. Xu, Scr. Mater. 64 (2011) 785.

[9] C.A. Schuh, T.C. Hufnagel, U. Ramamurty, Acta Mater. 55 (2007) 4067.

[10] M.D. Demetriou, J.C. Hanan, C. Veazey, M.D. Michiel, N. Lenoir, E. Üstündag, W.L Johnson, Adv. Mater. 19 (2007) 1957.

[11] M.D. Demetriou, J.P. Schramm, C. Veazey, W.L. Johnson, Appl. Phys. Lett. 91 (2007) 161903.

[12] M.D. Demetriou, C. Veazey, J.S. Harmon, J.P. Schramm, W.L. Johnson, Phys. Rev. Lett. 101 (2008) 145702.

[13] T. Wada, A. Inoue, Mater. Trans. 45 (2004) 2761.

[14] A.H. Brothers, D.C. Dunand, Scr. Mater. 54 (2006) 513.

[15] M.D. Demetriou, G. Duan, C. Veazey, K.D. Blauwe, W.L. Johnson, Scr. Mater. 57 (2007) 9.

[16] X. Wei, J.H. Chen, L.H. Dai, Scr. Mater. 66 (2012) 721.

[17] A.H. Brothers, D.C. Dunand, Acta Mater. 53 (2005) 4427

[18] A.H. Brothers, D.C. Dunand, Appl. Phys. Lett. 84 (2004) 1108.

[19] A.H. Brothers, D.C. Dunand, Q. Zheng, J. Xu, J. Appl. Phys. 102 (2007) 023508.

[20] M.E. Cox, S.N. Mathaudhu, K.T. Hartwig, D.C. Dunand, Metall. Mater. Trans. A 41 (2010) 1706

[21] M. Kiser, M.Y. He, F.W. Zok, Acta Mater. 47 (1999) 2685

[22] G.H. Wu, Z.Y. Dou, D.L. Sun, L.T. Jiang, B.S. Ding, B.F. He, Scr. Mater. 56 (2007) 221

[23] A. Mortensen, Concise Encyclopedia of Composite Materials, second ed. Elsevier Ltd, 2007.

[24] M. Kouzeli, A. Mortensen, Acta Mater. 50 (2002) 39.

25] A. Cohades, A. Mortensen, Acta Mater. 71 (2014) 39.

[26] A. Miserez, R. Müller, A. Rossoll, L. Weber, A. Mortensen, Mater. Sci. Eng. A 387 (2004) 822.

27] A. Mortensen, I. Jin, Int. Mater. Rev. 37 (1992) 101.

[28] H. Wang, R. Li, Y. Wu, X.M. Chu, X.J. Liu, T.G. Nieh, Z.P. Lu, Compos. Sci. Technol. 75 (2013) 49

[29] A. Peker, W.L. Johnson, Appl. Phys. Lett. 63 (1993) 2342.

[30] W. Chen, Z. Liu, H.M. Robinson, J. Schroers, Acta Mater. 73 (2014) 259.

[31] H. Choi-Yim, R. Busch, U. Koster, W.L. Johnson, Acta Mater. 47 (1999) 2455.

[32] S. Kadambi, K. Pramoda, U. Ramamurty, C.N.R. Rao, ACS Appl. Mater. Interfaces 7 (2015) 17016.

[33] P. Tandaiya, R. Narasimhan, U. Ramamurty, Acta Mater. 61 (2013) 1558.

[34] R. Bhowmick, R. Raghavan, K. Chattopadhyay, U. Ramamurty, Acta Mater. 54 (2006) 4221.

[35] X. Xi, D.Q. Zhao, W.H. Wang, Y. Wu, J.J. Lewandowski, Phys. Rev. Lett. 94 (2005) 125510. 